\title{
Bioanalytical Validation Method
}

National Cancer Institute

\section{Source}

National Cancer Institute. Bioanalytical Validation Method. NCI Thesaurus. Code

C115542.

A process that ensures the use of selective, sensitive, and validated analytical methods for the quantitative evaluation of drugs and their metabolites (analytes) and biomarkers, for the successful conduction of nonclinical and/or clinical trials. 